\title{
An empirical temperature calibration for the $\Delta a$ photometric system
}

\section{The A-type and mid F-type stars ${ }^{\star}$}

\author{
E. Paunzen, A. Schnell, and H. M. Maitzen
}

\author{
Institut für Astronomie der Universität Wien, Türkenschanzstr. 17, 1180 Wien, Austria \\ e-mail: Ernst.Paunzen@univie.ac.at
}

Received 21 January 2006 / Accepted 22 June 2006

\begin{abstract}
Context. With the $\Delta a$ photometric system, it is possible to study very distant galactic and even extragalactic clusters with a high level of accuracy. This can be done with a classical color-magnitude diagram and appropriate isochrones. The new calibration presented in this paper is a powerful extension.

Aims. For open clusters, the reddening is straightforward for an estimation via Isochrone fitting and is needed in order to calculate the reddening-free, temperature sensitive, index $\left(g_{1}-y\right)_{0}$. As a last step, the calibration can be applied to individual stars.

Methods. Because no a priori reddening-free photometric parameters are available for the investigated spectral range, we have applied the dereddening calibrations of the Strömgren $u v b y \beta$ system and compared them with extinction models for the Milky Way. As expected from the sample of bright stars, the extinction is negligible for almost all objects. As a next step, already established calibrations within the Strömgren $u v b y \beta$, Geneva 7-color, and Johnson $U B V$ systems were applied to a sample of 282 normal stars to derive a polynomial fit of the third degree for the averaged effective temperatures to the individual $\left(g_{1}-y\right)_{0}$ values.

Results. We derived an empirical temperature calibration for the $\Delta a$ photometric system for A-type to mid F-type with a mean of the error for the whole sample of $\Delta T_{\text {eff }}$ is $134 \mathrm{~K}$, which is lower than the value in Paper I for hotter stars. No statistically significant effect of the rotational velocity on the precision of the calibration was found.

Conclusions. We have derived a new intrinsically consistent, empirical, effective temperature calibration for a spectral range from early B-type to mid F-type, luminosity class V to III stars within the photometric $\Delta a$ system. The statistical mean error over the complete spectral range of about 140 to $240 \mathrm{~K}$ will allow to individual objects of far distant galactic be studied as well as extragalactic clusters with high accuracy.
\end{abstract}

Key words. stars: early-type - techniques: photometric

\section{Introduction}

We present the continuation of our efforts to derive an empirical temperature calibration for the photometric $\Delta a$ system (characteristics described in Paunzen et al. 2005b). In the first paper (Paunzen et al. 2005d, Paper I), the results for the B-type stars and the applied methods were published. In total, 225 stars were used to derive effective temperatures within the Strömgren uvby $\beta$, Geneva 7-color, and Johnson $U B V$ systems based on $(u-b), X$, and $(B-V)_{0}$, respectively. The final calibration for $\left(g_{1}-y\right)_{0}$ in the $\Delta a$ photometric system is valid for effective temperatures between 33000 and $10000 \mathrm{~K}$ and yields a statistical mean error of $238 \mathrm{~K}$ for the whole spectral range.

In this paper we investigate the A-type to mid F-type objects that exhibit an increase line blanketing and luminosity effects without the availability of any a priori reddening-free parameter. Only Strömgren $\beta$ does, in general, not depend on the extinction. However, it is sensitive not only to the effective temperature alone but also to the luminosity (Gerbaldi et al. 1999). Based on the methods used in Paper I, the following parameters were used for our purpose: $(b-y)_{0},(B 2-V 1)_{0}$, and $(B-V)_{0}$. The scatter of the derived effective temperatures for spectral types between $\mathrm{A} 0$

* Table 1 is only available in electronic form at the CDS via anonymous ftp to cdsarc.u-strasbg.fr $(130.79 .128 .5)$ or via http://cdsweb.u-strasbg.fr/cgi-bin/qcat?J/A+A/458/293 and A3 is not larger than that for later type stars because our sample of bright galactic-field stars is almost free of reddening.

Applying the same selection criteria as in Paper I yields 282 luminosity class V to III A-type to mid F-type stars. The derived mean errors for the effective temperature calibrations within all photometric systems are smaller than those from Paper I.

With the established intrinsically consistent, empirical, effective temperature calibrations for B-type to mid F-type stars, it is now possible to study individual objects in very distant galactic open clusters (Paunzen et al. 2005c) and extragalactic systems (Paunzen et al. 2005a) for which, in general, no photometric data are available within a standard system.

\section{Sample of program stars}

The selection criteria for the A-type to mid F-type objects are exactly the same as in Paper I and will not be repeated here. The Johnson, Geneva and Strömgren colors are excerpted from the General Catalogue of Photometric Data (GCPD, http://obswww. unige.ch/gcpd/gcpd.html, Mermilliod et al. 1997). The $\Delta a$ photometry is from the paper by Vogt et al. (1998).

The final list (Table 1) includes 282 objects and is only available in electronic form at the CDS or upon request from the first author. This table includes the identification of objects, 

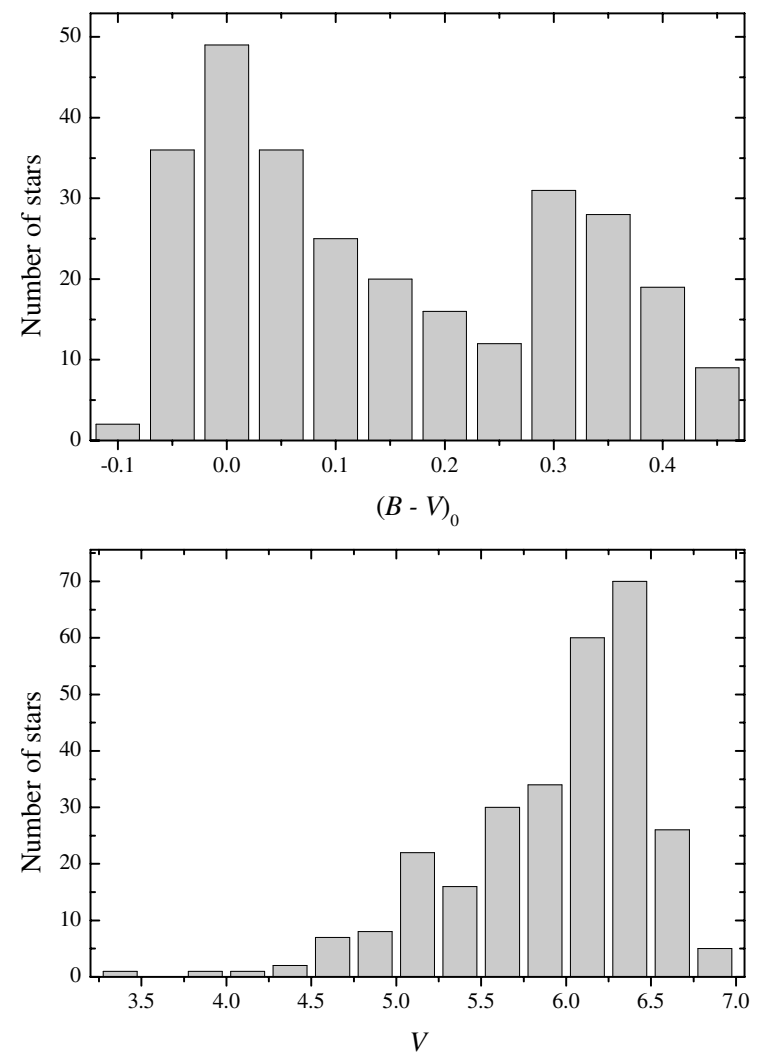

Fig. 1. The distribution of Johnson $(B-V)_{0}$ (upper panel) and $V$ (lower panel) for our sample of 282 main sequence A to mid F-type objects. The distribution of $(B-V)_{0}$ shows two maxima at spectral types of about $\mathrm{A} 0$ and $\mathrm{F} 0$.

the complete Strömgren $u v b y \beta,\left(g_{1}-y\right)_{0},(B-V)_{0}$, and $(B 2-V 1)_{0}$ values, $V$ magnitudes, $E(b-y)$ values, effective temperature with the corresponding errors, $v \sin i$ values, and spectral types, respectively.

The distribution of Johnson $V$ and $(B-V)_{0}$ for our sample is shown in Fig. 1. The estimation of the reddening is explained in more detail in Sect. 3. The distribution of $V$ is comparable to the one published in Paper I, peaking at $V=6.25$, mag, whereas the one for $(B-V)_{0}$ exhibits two maxima at spectral types of about A0 (+0.0 mag) and F0 (+0.3 mag, see also Table 2). However, there is a statistically significant number of objects in the complete investigated spectral range.

\section{Estimation of the reddening}

Our sample comprises stars later than a spectral type of A0. A typical A0 main sequence object has a mean absolute magnitude of about +0 mag. A visual magnitude of 7 mag (Fig. 1) then corresponds to a distance of $250 \mathrm{pc}$, for which the reddening can be almost neglected in all directions (Neckel et al. 1980).

The following photometric calibrations in the Strömgren $u v b y \beta$ system were used to estimate the reddening according to the valid spectral range that is estimated by the standard relations of the different indices:

- A0 - A3: Crawford (1978) and Hilditch et al. (1983);

- A3 - F0: Crawford (1979) and Domingo \& Figueras (1999);

- later than F0: Crawford (1973) and Schuster \& Nissen (1989).

The calibrations are not very reliable for stars with spectral types between A0 and A3 (Gerbaldi et al. 1999), mainly because for these stars, the reddening-free parameter $\beta$ is no longer a temperature indicator alone but is also sensitive to the luminosity, therefore the standard relation of $(b-y)_{0}$ versus $m_{0}$ as listed by Hilditch et al. (1983) and their method of calibration were used. Only some objects that fall outside the given relation were dereddened using the calibration by Crawford (1978).

Furthermore, we used the interstellar extinction model by Chen et al. (1998) to derive the reddening for all program stars as described in Paper I. The values from the calibration of the Strömgren $u v b y \beta$ and the model by Chen et al. (1998) very closely agree. As expected, all objects have a calibrated total absorption $A_{\mathrm{V}}$ of less than 0.35 mag with 233 (82\% of the complete sample) stars even lower than $0.05 \mathrm{mag}$. Taking the following relations into account:

$$
\begin{aligned}
A_{\mathrm{V}} & =3.1 E(B-V)=4.3 E(b-y)=4.95 E(B 2-V 1) \\
& =7.95 E\left(g_{1}-y\right),
\end{aligned}
$$

the effect of the reddening on the calibration for our sample can be neglected and does not introduce a significant error source.

\section{The calibration of the effective temperature}

As in Paper I, the first step was to derive the effective temperature for each individual star within the Geneva, Strömgren, and Johnson photometric systems. For this purpose we used the reddening relations as listed in Eq. (1) to calculate the unreddened indices that are necessary to make the proper calibration as listed.

Geneva system: the calibration by Künzli et al. (1997) is the most recent for the investigated spectral range. For intermediate stars hotter than $8500 \mathrm{~K}$, they use the parameters $p T$ and $p G$, which are linear combinations of the seven Geneva colors. Those two indices can be dereddened with the following relations: $p T_{0}=p T-E(B 2-V 1)$ and $p G_{0}=p G-1.1 E(B 2-V 1)$. For cooler objects, the grids of $m_{2}$ and $d$ versus $(B 2-V 1)_{0}$ serve as a calibration tablet. The definition of these indices are listed in Golay (1994).

Strömgren system: Napiwotzki et al. (1993) investigated several calibrations based on $a_{0}$ and $r^{*}$ for hotter, as well as $\beta$, and $c_{0}$ for cooler objects yielding a rather unsatisfactory result. Finally, they established a $T_{\text {eff }}$ versus $(b-y)_{0}$ relation (Eq. (10) therein), which was applied to our sample.

Johnson system: we used the semi-empirical $T_{\text {eff }}$ versus $(B-V)_{0}$ relation as listed in Gray (1992; Eq. (15.14)). It is based on synthetic colors from theoretical stellar atmospheres that are normalized to observations of spectroscopic binary systems, as well as bright stars. It is given as

$$
\begin{aligned}
\log T_{\text {eff }}= & +3.988-0.881(B-V)_{0}+2.142(B-V)_{0}^{2} \\
& -3.614(B-V)_{0}^{3}+3.2637(B-V)_{0}^{4} \\
& -1.4727(B-V)_{0}^{5}+0.2600(B-V)_{0}^{6}
\end{aligned}
$$

and is valid for all A-type to mid F-type, luminosity class III to V objects. It superseded the relation listed in Code et al. (1976).

The individual effective temperature values for the three photometric systems were first tested for their intrinsic consistency and then averaged. The final values, together with the standard deviations of the means, are listed in the Table 1 which is electronically-available. No statistical significant outliers in any photometric system were detected. 


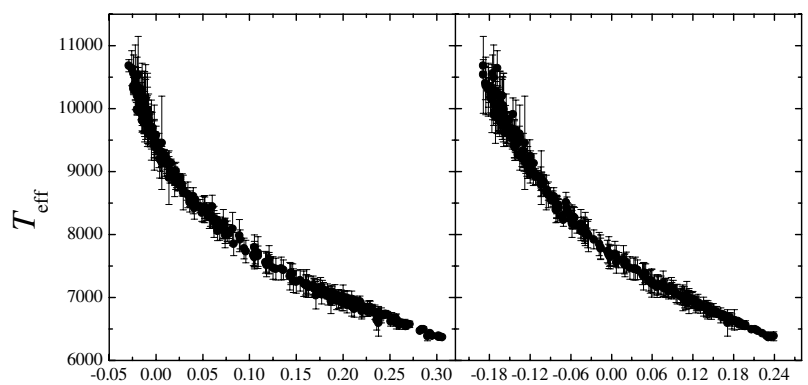

$(b-y)_{0}$

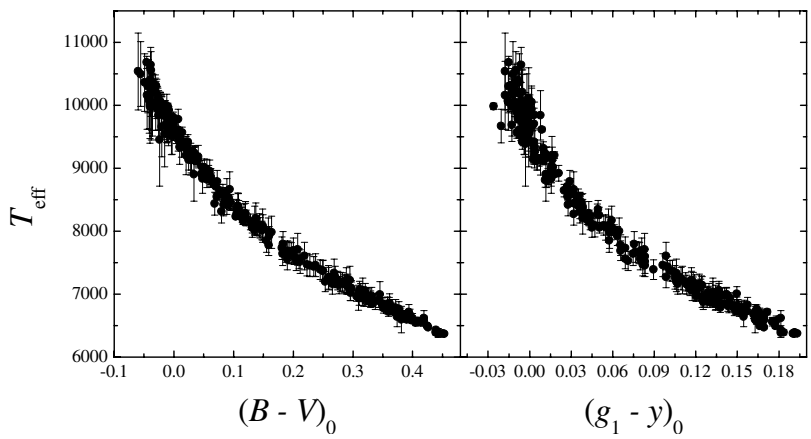

Fig. 2. Mean relation between the effective temperature and $(b-y)_{0}$, $(B 2-V 1)_{0},(B-V)_{0}$, as well as $\left(g_{1}-y\right)_{0}$ for A-type to mid F-type, luminosity class $\mathrm{V}$ to III objects.

\section{The result for the $\Delta a$ photometric system}

The relation between the mean effective temperature and the different temperature sensitive indices for the four investigated photometric systems is shown in Fig. 2. The errors are constant over the complete spectral range. There is a larger scatter only for the Geneva $(B 2-V 1)_{0}$ index at effective temperatures hotter than $9000 \mathrm{~K}$. We checked this region with the grids of $p T_{0}$ and $p G_{0}$ originally used. It is exactly where both indices become zero for the Main Sequence and small shifts result in larger uncertainties. It therefore seems to be an intrinsic numerical problem of the grids themselves; however the overall statistical error of $(B 2-V 1)_{0}$ is satisfying. The final calibrations parameterized as third-degree polynomials are:

$$
\begin{aligned}
\log T_{\mathrm{eff}}= & +3.9793(4)-1.34(2) \cdot(b-y)_{0} \\
& +4.90(15) \cdot(b-y)_{0}^{2}-8.06(38) \cdot(b-y)_{0}^{3} \\
= & +3.8853(4)-0.459(5) \cdot(B 2-V 1)_{0} \\
& +1.02(2) \cdot(B 2-V 1)_{0}^{2} \\
& -2.36(16) \cdot(B 2-V 1)_{0}^{3} \\
= & +3.9825(4)-0.670(10) \cdot(B-V)_{0} \\
& +1.05(7) \cdot(B-V)_{0}^{2}-0.98(12) \cdot(B-V)_{0}^{3} \\
= & +3.9817(8)-1.79(5) \cdot\left(g_{1}-y\right)_{0} \\
& +8.14(77) \cdot\left(g_{1}-y\right)_{0}^{2}-19(3) \cdot\left(g_{1}-y\right)_{0}^{3}
\end{aligned}
$$

with the mean of the errors for the whole sample of $\Delta T_{\mathrm{eff}}[(b-$ $\left.y)_{0},(B 2-V 1)_{0},(B-V)_{0},\left(g_{1}-y\right)_{0}\right]=[66,100,71,134 \mathrm{~K}]$. These are statistical errors for the complete sample. The errors for individual stars are, of course, larger than that. Note that the error for the Johnson $(B-V)_{0}$ calibration is surprisingly small. It shows the overall robustness of broad-band indices against metallicity and luminosity effects, which makes them superior for statistical analysis of larger samples over a wide spectral range. However, it has to be emphasized that it is a priori not possible to apply
Table 2. Mean relation between the effective temperature and $(B-V)_{0}$, $(b-y)_{0},(B 2-V 1)_{0}$, as well as $\left(g_{1}-y\right)_{0}$ for A-type to mid F-type, luminosity class V to III objects. Only spectral types according to the Yerkes system (Keenan 1985) are listed.

\begin{tabular}{crcccc}
\hline \hline Spec. & $T_{\text {eff }}$ & $(B-V)_{0}$ & $(b-y)_{0}$ & $(B 2-V 1)_{0}$ & $\left(g_{1}-y\right)_{0}$ \\
\hline A0 & 10000 & -0.025 & -0.015 & -0.160 & -0.010 \\
A2 & 8750 & +0.067 & +0.031 & -0.093 & +0.025 \\
A3 & 8300 & +0.112 & +0.055 & -0.060 & +0.042 \\
A5 & 7900 & +0.161 & +0.082 & -0.024 & +0.062 \\
A7 & 7500 & +0.221 & +0.120 & +0.022 & +0.087 \\
F0 & 7050 & +0.304 & +0.185 & +0.087 & +0.125 \\
F2 & 6750 & +0.368 & +0.238 & +0.141 & +0.155 \\
F3 & 6650 & +0.390 & +0.254 & +0.159 & +0.165 \\
F5 & 6450 & +0.433 & +0.284 & +0.196 & +0.184 \\
\hline
\end{tabular}

dereddening methods in the Johnson $U B V$ system for stars cooler than B9 (Johnson 1958).

The statistical error for $\left(g_{1}-y\right)_{0}, 134 \mathrm{~K}$ is significantly smaller than the one for the hotter stars $(238 \mathrm{~K})$. But one has to keep in mind that the absolute errors are over the complete spectral range from early B-type to F-type constant, but only the relative one, as listed, is decreasing.

Table 2 lists the mean relations between the effective temperature and $(B-V)_{0},(b-y)_{0},(B 2-V 1)_{0}$, as well as $\left(g_{1}-y\right)_{0}$, depending on spectral types (taken from Paunzen et al. 2005b). Only standard spectral types of the Yerkes system (Keenan 1985) are given. The $(B-V)_{0}$ and $\left(g_{1}-y\right)_{0}$ values for $\mathrm{A} 0$ are, within the error limits, identical to those derived in Paper I (Table 1), which guarantees an intrinsic consistent calibration from B0 to F5 for the $\Delta a$ photometric system.

As in Paper I, we checked the effect of high $v \sin i$ objects on the derived calibrations in the same manner. This might be an issue for early A-type objects only because the rotational velocities decrease significantly for cooler objects (Fekel et al. 2004). Again, no statistical significant effect of the rotational velocity, which can be distinguished from other error sources, was found on the precision of the calibration.

The detailed calibration procedure for determining the effective temperature within the $\Delta a$ photometric system is given in Paper I. We only give a short overview of the main method here. After the estimation of the reddening $E\left(g_{1}-y\right)$, the dereddened standard $\left(g_{1}-y\right)_{0}$ has to be calculated and the calibration for the appropriate spectral region applied. As a final check, a comparison with the values for other photometric systems, if available, should be performed.

\section{Conclusion}

In the second and last parts of this series, we established an empirically effective temperature calibration for the $\Delta a$ photometric system for main sequence (luminosity class V to III) A-type to mid F-type star. Applying the same methods as in Paper I, the calibrations in the Strömgren $u v b y \beta$, Geneva 7-color, and Johnson $U B V$ photometric systems based on $(b-y)_{0},(B 2-V 1)_{0}$, and $(B-V)_{0}$, respectively are used to derive effective temperature for 282 normal type objects. The final calibrations are expressed third-degree polynomials.

As expected from the brightness and thus the distance from the Sun $(<250 \mathrm{pc})$ for our sample, the reddening can almost be neglected for most of the objects. This is important because no reddening-free photometric parameter is a priori available for the whole investigated spectral domain. 
The established standard system guarantees a direct link to the calibration for B-type stars published in Paper I. It is therefore possible to calibrate effective temperatures via $\left(g_{1}-y\right)_{0}$ in a consistent way for spectral types from B0 to F5 for luminosity classes V to III with a constant absolute error of a few percent.

This will allow us to independently investigate individual stars of distant galactic, as well as extragalactic open clusters within the $\Delta a$ photometric system, to a high level of accuracy.

Acknowledgements. This research was performed within the projects $P 17580$ and P17920 of the Austrian Fonds zur Förderung der wissenschaftlichen Forschung (FwF). Use was made of the SIMBAD database, operated at the CDS, Strasbourg, France, and of the NASA's Astrophysics Data System. We would like thank the referee for her/his important comments pointing at serious problems in the first version.

\section{References}

Chen, B., Vergely, J. L., Valette, B., \& Carraro, G. 1998, A\&A, 336, 137 Code, A. D., Bless, R. C., Davis, J., \& Brown, R. H. 1976, ApJ, 203, 417 Crawford, D. L. 1975, AJ, 80, 955

Crawford, D. L. 1978, AJ, 83, 48

Crawford, D. L. 1979, AJ, 84, 1858
Domingo, A., \& Figueras, F. 1999, A\&A, 343, 446

Fekel, F. C., Warner, P. B., \& Kaye, A. B. 2004, in Stellar Rotation, ed. A. Maeder, \& Ph. Eenens, San Francisco, ASP Conf. Ser., 215, 53

Gerbaldi, M., Faraggiana, R., Burnage, R., et al. 1999, A\&AS, 137, 273

Golay, M. 1994, in The MK process at 50 years. A powerful tool for astrophysical insight, ed. Ch. Corbally, R. O. Gray, \& R. F. Garrison, San Francisco, ASP Conf. Ser., 60, 164

Gray, D. F. 1992, The Observation and Analysis of Stellar Photospheres (Cambridge University Press)

Hilditch, R. W., Hill, G., \& Barnes, J. V. 1983, MNRAS, 204, 241

Johnson, H. L. 1958, Lowell Obs. Bull., 4, 37

Keenan, P. C. 1985, in Calibration of fundamental stellar quantities (Dordrecht: D. Reidel Publishing Co.), 121

Künzli, M., North, P., Kurucz, R. L., \& Nicolet, B. 1997, A\&AS, 122, 51

Mermilliod, J.-C., Mermilliod, M., \& Hauck, B. 1997, A\&AS, 124, 349

Napiwotzki, R., Schönberner, D., \& Wenske, V. 1993, A\&A, 268, 653

Neckel, Th., Klare, G., \& Sarcander, M. 1980, A\&AS, 42, 251

Paunzen, E., Pintado, O. I., Maitzen, H. M., \& Claret, A. 2005a, MNRAS, 362, 1025

Paunzen, E., Stütz, Ch., \& Maitzen, H. M. 2005b, A\&A, 441, 631

Paunzen, E., Netopil, M., Iliev, I. Kh., et al. 2005c, A\&A, 443, 157

Paunzen, E., Schnell, A., \& Maitzen, H. M. 2005d, A\&A, 444, 941 (Paper I)

Schuster, W. J., \& Nissen, P. E. 1989, A\&A, 221, 65

Vogt, N., Kerschbaum, F., Maitzen, H. M., \& Faúndez-Abans, M. 1998, A\&AS, 130,455 